\title{
Clopidogrel use after Spinal Cord Stimulator Implantation
}

To the Editor:

Regarding your recent review of bleeding risk in interventional pain practice, we noted there was no reference to the safety of initiating or maintaining clopidogrel, a thienopyridine derivative, in patients for whom spinal cord stimulators (SCS) have already been implanted (1). An extensive Medline search revealed that this question has yet to be addressed in the literature. An ever increasing number of patients will be requiring hemostasis altering drugs as vascular disease remains the leading cause of death in the United States and Europe.

The insertion of epidural and subarachnoid needles and catheters in patients taking hemostasis-altering medication runs the risk of spinal cord hematoma and resulting compression. The incidence of spinal hematoma following epidural injection is estimated to be $<1$ in 150,000 to $<1$ in 220,000 spinal anesthetics (2). Is the risk so low as to make randomized controlled trials difficult to perform? What then do physicians use as guidelines - case reports, consensus boards, and clinical judgment on individual patients?

There are large number of patients who have or would benefit from a spinal cord stimulator who also have atherosclerotic disease and aspirin sensitivity. This issue will only become more common as the number of SCS increases. Isolated use of aspirin and non-steroidal anti-inflammatory medications appear to have limited risk for development of bleeding complications with neuraxial procedures (3). This is not known for thienopyridine derivatives and studies have not been done for SCS with NSAIDs and aspirin.

Currently, the American Society for Regional Anesthesia (ASRA) states that the risk of spinal hematoma with neuraxial procedures is unknown. There are recommendations stating that thienopyridine derivates be stopped 7-14 days pri- or to neuraxial procedures (4). These recommendations are for safety and are not based on clinical trials. The available data are very limited. What about patients who have a successfully functioning SCS and then develop coronary artery stenosis requiring a stent or any of a number of other vascular diseases? After stent placement most cardiology literature states clopidogrel should be used for twelve months (5). Is this safe for patients in whom there is an SCS?

At our clinic we have a 61-year-old male with spinal stenosis and radicular signs who received excellent relief from his SCS placed three years ago. He was lost to follow up for sixteen months and returned to our clinic when his internal pulse generator (IPG) had failed due to expired battery life. During that time he had a coronary artery stent placed for progressive angina and was placed on clopidogrel $75 \mathrm{mg}$ per day for approximately eleven months. The battery was changed without complication. However, we were concerned that if SCS lead migration, fracture or damage occurred while he was on a thienopyridine, there may be an increased risk of epidural hematoma. After discussion with the patient and his cardiologist, it was decided that the clopidogrel could safely be discontinued because he was two weeks away from completing his post-stent antiplatelet therapy course. The clopidogrel was discontinued but the question remains.

Given the paucity of data and the very low incidence of spinal hematoma with neuraxial procedures in general, would it be prudent to establish an anonymous database of complications regarding the use of clopidogrel in patients with SCS? The risk of hematoma may be negligible, but does exist. Pain physicians should consider the risks and benefits of clopidogrel in all patients with coexisting atherosclerotic disease who undergo SCS placement.

\section{References}

1. Raj PP, Shah RV, Kaye AD et al. Bleeding risk in interventional pain practice: assessment, management, and review of the literature. Pain Physician 2004; 7:3-51.

2. Tryba M. Rückmarksnahe regionalalanästhesie und niedermolekukare heparine: pro. Anasthesiol Intensivmed Notfallmed Schmerzther 1993; 28:179-181.

3. Horlocker TT, Bajwa ZH, Ashraf Z et al. Risk assessment of hemorrhagic complications associated with nonsteroidal anti-inflammatory medications in ambulatory pain clinic patients undergoing epidural steroid injection. Anesth Analg 2002;95:16911697.

4. Second Consensus Conference on Neuraxial Anesthesia and Anticoagulation. April 25-28, 2002. or Second Consensus Conference on Neuraxial Anesthesia and Anticoagulation. Reg Anesth Pain Med 2003; 28:172-179.

5. Berger PB, Bell MR, Rihal CS. Clopidogrel versus ticlopidine after intracoronary stent placement. J Am Coll Cardiol 1999; 34: 1891-1894

\section{George Veech, MD}

Fellow in Pain Medicine

Harvard Medical School

Massachusetts General Hospital

55 Fruit Street

Boston, MA 02114

E-mail: gveech@partners.org

Steven A. Barna, MD

Instructor of Anesthesia

Harvard Medical School

Medical Director of Pain Clinic

Massachusetts General Hospital

55 Fruit Street

Boston, MA 02114

E-mail: sbarna@partners.org

Milan P. Stojanovic, MD

Assistant Professor of Anesthesia

Harvard Medical School

Director of Interventional Pain

Program

Massachusetts General Hospital

55 Fruit Street

Boston, MA 02114

E-mail: mstojanovic@partners.org 